\title{
Detección molecular de Rickettsia massiliae y Anaplasma platys en garrapatas Rhipicephalus sanguineus y caninos domésticos del municipio de Bahía Blanca (Argentina)
}

\section{Gabriel L. Cicuttin, Pablo Vidal, M. Nazarena De Salvo, Fernando J. Beltrán y Federico E. Gury Dohmen}

\section{Molecular detection of Rickettsia massiliae and Anaplasma platys infecting Rhipicephalus sanguineus ticks and dogs, Bahía Blanca (Argentina)}

Background: Rickettsioses, ehrlichioses and anaplasmoses are caused by Gram negative obligate intracellular bacteria and transmitted mainly by arthropods. Aim: To detect and perform the molecular characterization of these pathogens in ticks and domestic dogs in Bahia Blanca City (Buenos Aires, Argentina). Methods: Fifty six blood samples from dogs and 82 ticks (75 Rhipicephalus sanguineus and 7 Amblyomma tigrinum) were studied. The samples were analyzed by PCR for Rickettsia (intergenic space 23S-5S rRNA), Ehrlichia/Anaplasma (16S rRNA), and Anaplasma platys (16S rRNA). Results: $12 \%$ of $R$. sanguineus resulted positive for Rickettsia, identified by sequencing as Rickettsia massiliae; and $37.5 \%$ of the canine blood samples analyzed were positive for $A$. platys. Molecular characterization was also performed by amplification of the fragment of the citrate synthase gene (gltA) (Rickettsia genus) and the groESL gene (A. platys). Phylogenetic trees were constructed using the neighbor-joining method. These trees revealed that sequences obtained are similar to those from other geographical regions. Conclusion: The results indicate the presence of R. massiliae in R. sanguineus ticks for the second time in an urban area of South America and A. platys infection in dogs, being the southernmost region of Argentina where it has been notified.

Key words: Rickettsia massiliae, Anaplasma platys, Rhipicephalus sanguineus, dogs, South America.

Palabras clave: Rickettsia massiliae, Anaplasma platys, Rhipicephalus sanguineus, perros, Sudamérica.

\section{Introducción}

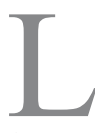

as rickettsiosis, ehrlichiosis y anaplasmosis son causadas por bacterias gramnegativas, intracelulares obligadas, pertenecientes al orden Rickettsiales (género Rickettsia, familia Rickettsiaceae; géneros Ehrlichia y Anaplasma, familia Anaplasmataceae $)^{1}$, transmitidas principalmente por artrópodos. Son consideradas enfermedades emergentes en humanos y animales ${ }^{2,3}$.

En los últimos tiempos se ha registrado un incremento en la ocurrencia de casos humanos de rickettsiosis en áreas urbanas. Es así que emergieron nuevos patógenos humanos como $R$. massiliae y $R$. felis, mientras que otras rickettsiosis tradicionalmente relacionadas con áreas silvestres (tales como $R$. rickettsii y $R$. parkeri) comenzaron a diseminarse a estas áreas a medida que aumentaba la dispersión de distintos vectores y reservorios, generalmente a causa de las acciones humanas. Además se encontraron nuevos ciclos de transmisión como en el caso de $R$. typh $i$, incluyendo a animales de compañía y sus ectoparásitos ${ }^{4}$.

Rickettsia massiliae, perteneciente al grupo de las fiebres manchadas, se encuentra en distintas regiones del mundo, asociada a garrapatas del género Rhipicephalus (incluyendo la garrapata común del perro Rhipicephalus sanguineus $)^{4,5}$. Durante muchos años, la patogenicidad de R. massiliae fue desconocida, siendo identificada como patógeno humano por primera vez de forma retrospectiva en 2005, a partir de muestras de un paciente con rickettsiosis acaecida en la década del $80^{6}$. Se han confirmado pocos casos de enfermedad en el hombre, aunque $R$. massiliae ha sido implicada en numerosos casos europeos por métodos serológicos ${ }^{5-8}$. Los signos clínicos incluyen fiebre, cefalea, lesión necrótica en el sitio de picadura de la garrapata, erupción purpúrica maculo-papular en extremidades, espalda, palmas de las manos y plantas de los pies, y también afección ocular con disminución aguda bilateral de la visión asociada a corio-retinitis ${ }^{4,5,7,8}$. En caninos domésticos también podría causar enfermedad, con signos similares a otras rickettsiosis caninas, tales como fiebre, anorexia, mialgias, signos neurológicos, presencia de edema e hiperemia en labios, prepucio, escroto, orejas y extremidades, y lesiones oculares ${ }^{4,9}$.

Anaplasma platys presenta distribución mundial y se transmite por garrapatas $R$. sanguineus, es el agente de la trombocitopenia cíclica infecciosa canina, afección generalmente leve, aunque con diversa patogenicidad
Instituto de Zoonosis Luis Pasteur, Ciudad Autónoma de Buenos Aires -CABA- Argentina. Sección Serología y Pruebas Biológicas (FEGD). Área Zoonosis Bacterianas y Parasitarias Transmitidas po Vectores (GLC, NDSM) Área Zoonosis Virales. (FJB). Municipalidad de Bahía Blanca, Provincia de Buenos Aires, Argentina

Dirección de Zoonosis (PV).

Proyecto parcialmente subsidiado por el Subsidio para investigación médica en Medicina sanitaria y epidemiología (2010) de la Fundación Alberto J. Roemmers.

Recibido: 11 de abril de 2014 Aceptado: 25 de junio de 2014

\section{Correspondencia a:}

Gabriel L. Cicuttin gcicuttin@gmail.com 
en distintas regiones. La enfermedad puede cursar con fiebre, anorexia, petequias, uveítis, linfoadenopatía generalizada, leucopenia, anemia moderada y especialmente trombocitopenia, que ocurre en episodios de 3-4 días y a intervalos de 7-21 días, dando paso con el tiempo a una trombocitopenia crónica de lenta recuperación ${ }^{10-12}$. En cuanto al rol zoonótico de $A$. platys, los datos de estudios actuales disponibles no son concluyentes ${ }^{10,13}$.

En Sudamérica, se han detectado distintas rickettsias (Rickettsia rickettsii, $R$. parkeri, $R$. massiliae y $R$. felis, entre otras) y ehrlichias (Ehrlichia chaffeensis y E. canis) asociadas a cuadros clínicos en humanos. También se describieron otras especies en garrapatas y en diferentes hospederos $^{14-16}$.

En Argentina, este grupo de enfermedades fue poco estudiado en zonas urbanas; por ello, el objetivo del presente trabajo fue estudiar la presencia de rickettsias, ehrlichias y anaplasmas en garrapatas y caninos domésticos procedentes en un área urbana como es el municipio de Bahía Blanca (sur de la provincia de Buenos Aires, Argentina), y caracterizar molecularmente los hallazgos obtenidos.

\section{Material y Métodos}

\section{Muestras}

Entre noviembre de 2009 y febrero de 2011 se recolectaron garrapatas y sangre completa de caninos domésticos (Canis familiaris) procedentes del Municipio de Bahía Blanca $\left(38^{\circ} 43^{\prime} 0^{\prime}\right.$ 'S $\left.62^{\circ} 16^{\prime} 0^{\prime \prime} \mathrm{O}\right)$ en el sur de la provincia de Buenos Aires (Argentina), distante a más de $700 \mathrm{~km}$ del AMBA. Con más de 300.000 habitantes, es la tercera ciudad de la provincia de Buenos Aires, sin considerar la región metropolitana del Gran Buenos Aires. El emplazamiento geográfico de Bahía Blanca se caracteriza por una planicie que desciende hacia la línea costera. El clima es templado con una temperatura media anual de $15,5^{\circ}$ $\mathrm{C}$ y un registro anual de lluvias de 650 milímetros. La ciudad cuenta con una importante área urbana central. Los espacios verdes y barrios residenciales bordean el casco urbano, principalmente hacia los sectores norte y noroeste.

El estudio fue aprobado por el Comité de Docencia e Investigación del Instituto de Zoonosis Luis Pasteur del Ministerio de Salud del Gobierno de la Ciudad Autónoma de Buenos Aires y por la Dirección de Zoonosis de la Municipalidad de Bahía Blanca (Provincia de Buenos Aires, Argentina).

Los caninos fueron seleccionados al azar de animales clínicamente sanos que concurrieron al programa de esterilizaciones quirúrgicas organizado por el municipio. Las muestras sanguíneas fueron extraídas por punción de vena cefálica o yugular, con anticoagulante EDTA, bajo el consentimiento de los propietarios de los caninos. Las garrapatas se colectaron en forma manual de cada animal y se identificaron taxonómicamente siguiendo las claves descritas ${ }^{17}$. Todas las muestras se conservaron a $-70^{\circ} \mathrm{C}$ hasta su procesamiento.

\section{Extracción de ácidos nucleicos}

Las ninfas de garrapatas fueron reunidas en grupos de 4-5 ejemplares por perro y los adultos se extrajeron en forma individual. Cada ejemplar o grupo fue resuspendido en tampón Tris-EDTA, seccionado con bisturí y macerado. La extracción del ADN se realizó por el método del tiocianato de guanidina ${ }^{18}$.

El ADN de la sangre completa fue extraído con el AxyPrep Multisource Genomic DNA Miniprep Kit (Axygen Biosciences, EEUU), siguiendo las instrucciones del fabricante.

Se utilizó agua libre de nucleasas como control negativo de extracción.

\section{Amplificación}

La amplificación del género Rickettsia fue realizada con oligonucleótidos para una porción del espacio intergénico 23S-5S ARNr ${ }^{19}$. Para una mejor caracterización de las muestras positivas, se realizó una segunda reacción de polimerasa en cadena (RPC) para un fragmento del gen de la citrato sintasa ( $g l t A)$, siguiendo la metodología descrita por los autores ${ }^{20}$. Rickettsia. parkeri fue utilizada como control positivo.

Para la familia Anaplasmataceae se utilizaron oligonucleótidos para un fragmento de $16 \mathrm{~S} \mathrm{ARNr}^{21}$, utilizándose como control positivo A. bovis. A las muestras positivas se les realizó dos RPC específicas para $A$. platys: fragmento de $16 \mathrm{~S}$ ARNr y del gen groESL, según los procedimientos indicados por los autores ${ }^{22,23}$.

Como control negativo se usó agua libre de nucleasas.

\section{Secuenciación}

Los productos amplificados fueron purificados con PureLink ${ }^{\text {TM }}$ Quick Gel Extraction and PCR Purification Combo Kit ${ }^{\circledR}$ (Invitrogen-Life Technologies, Carlsbad, CA, EEUU) y secuenciados en un secuenciador 3500 Genetic Analyzer sequencer (Applied Biosystems, Foster City, CA, EEUU) en el Servicio de Neurovirosis del Instituto Nacional de Enfermedades Infecciosas (ANLIS Dr. Carlos G. Malbrán, Argentina). Las secuencias obtenidas fueron comparadas con secuencias disponibles en el GenBank, mediante la utilización del software BLAST (www.ncbi.nlm.nih.gov/blast).

\section{Análisis filogenético}

El análisis de alineamiento múltiple fue realizado con el algoritmo CLUSTAL y la construcción del árbol filogenético fue hecha con el software MEGA versión $5^{24}$. Para cada gen analizado, un filograma fue construido usando el método del vecino más cercano (neighbor-joining, NJ) y los parámetros del modelo Kimura. Los valores 
de confianza para cada rama de los árboles resultantes fueron determinados mediante 1.000 replicaciones de remuestreo (bootstrap).

\section{Números de acceso de las secuencias nucleotídicas}

Las secuencias obtenidas en este estudio fueron depositadas en la base de datos GenBank con los siguientes números de acceso: KF826281 (fragmento espacio intergénico 23S-5S de $R$. massiliae), KF826286 (fragmento del gen glt $A$ de $R$. massiliae), KF826282 y KF826284 (fragmento del $16 \mathrm{~S}$ ARNr de A. platys de los perros 17 y 103, respectivamente), KF826283 y KF826285 (fragmento del gen groESL de $A$. platys de los perros 17 y 103 , respectivamente).

\section{Resultados}

Se obtuvo muestra de 56 caninos domésticos parasitados con garrapatas. De estos animales se recolectaron 82 garrapatas, identificadas como pertenecientes a $R$. sanguineus (75 en total, nueve ninfas y 66 adultos) y Amblyomma tigrinum (7 adultos).

Género Rickettsia. La amplificación por RPC del espacio intergénico 23S-5S ARNr de Rickettsia spp., detectó positividad en nueve adultos de $R$. sanguineus colectados de sólo dos caninos (2/56). Todas las ninfas $R$. sanguineus, las garrapatas $A$. tigrinum y los caninos fueron negativos (Tabla 1).

De los productos positivos obtenidos de la RPC del espacio intergénico 23S-5S ARNr se logró secuenciar $6 / 9$ positivos. Las secuencias resultaron en $100 \%$ de identidad entre sí, así como con $R$. massiliae strain G83 Ciudad Autónoma de Buenos Aires -CABA-(KC525896), R. massiliae str. AZT80 (CP003319) y Rickettsia sp. Bar29 (AY125014), y 99,7\% con R. massiliae MTU5 (CP000683).

En la RPC del gen gltA sólo resultaron positivas 2/9 muestras positivas analizadas (Tabla 1), y ambas fueron secuenciadas. Las secuencias resultaron en 100\% con $R$. massiliae AZT80 (CP003319) y Rickettsia sp. Bar29 (U59720), y 99,9\% con Rickettsia sp. PoTiR600 (HM149282) y R. massiliae MTU5 (CP000683). El análisis filogenético del gen gltA por el método NJ se muestra en la Figura 1.

Familia Anaplasmataceae. En la RPC del gen $16 \mathrm{~S}$ ARNr de la familia Anaplasmataceae, 21 muestras de sangre canina resultaron positivas y de estos perros se estudiaron 35 garrapatas que resultaron negativas. Todas las garrapatas restantes fueron también negativas (Tabla 1).

Las 21 muestras positivas resultaron también positivas a las otras dos RPCs específicas para A. platys (16S ARNr y gen groESL). Es importante destacar que un perro fue positivo para $A$. platys, mientras que sus garrapatas resultaron positivas para $R$. massiliae.

\begin{tabular}{|c|c|c|c|c|c|c|}
\hline & \multirow{3}{*}{$\begin{array}{c}\text { Total } \\
\mathbf{n}\end{array}$} & \multicolumn{5}{|c|}{ n positivos/n testeados (\%) } \\
\hline & & \multicolumn{2}{|c|}{ Rickettsia spp. } & \multirow{2}{*}{$\begin{array}{c}\text { Familia } \\
\text { Anaplasmataceae } \\
\text { 16S ARNr }\end{array}$} & \multicolumn{2}{|c|}{ A. platys } \\
\hline & & $23 S-5 S$ & gltA & & 16S ARNr & groESL \\
\hline Complejo R. sanguineus & 75 & $\begin{array}{c}9 / 75 \\
\left(12,0^{\star}\right)\end{array}$ & $\begin{array}{c}2 / 9 \\
(22,2)\end{array}$ & $\begin{array}{c}0 / 75 \\
(0)\end{array}$ & - & - \\
\hline A. tigrinum & 7 & $\begin{array}{l}0 / 7 \\
(0)\end{array}$ & - & $\begin{array}{l}0 / 7 \\
(0)\end{array}$ & - & - \\
\hline Caninos domésticos & 56 & $\begin{array}{c}0 / 56 \\
(0)\end{array}$ & - & $\begin{array}{l}21 / 56 \\
(37,5)\end{array}$ & $\begin{array}{l}21 / 21 \\
(100)\end{array}$ & $\begin{array}{l}21 / 21 \\
(100)\end{array}$ \\
\hline
\end{tabular}

Se secuenciaron al azar ocho productos de la RPC A. platys $16 \mathrm{~S}$ ARNr, evidenciándose siete con $100 \%$ de identidad entre sí, así como con $A$. platys 83 CABA (KC525894), A. platys 165495 Buenos Aires (JX261979) y Ehrlichia platys Lara Venezuela (AF399917), entre otras. Por otra parte, la secuencia obtenida del perro identificado como 103 resultó con una identidad de 99,8\% con respecto al resto.

También se secuenciaron al azar tres productos de la RPC A. platys groESL (incluyendo el producto del perro 103). Todas las secuencias resultaron en $100 \%$ de identidad entre sí y con A. platys 83 CABA (KC525895), E. platys Lara Venezuela (AF399916), entre otras y 99,6\% con A. platys 17 Santiago de Chile (EF201806). El análisis filogenético del gen groESL por el método NJ se muestra en la Figura 2.

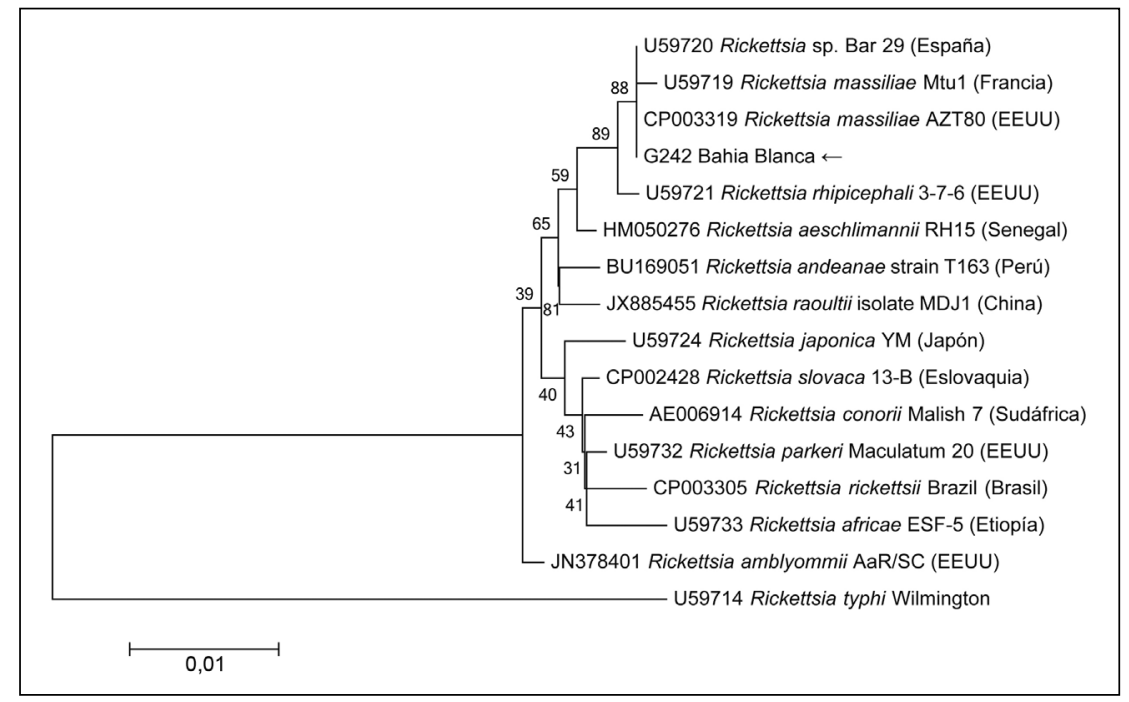

Figura 1. Árbol filogenético inferido por la comparación de secuencias parciales del gen gltA del género Rickettsia. Los números en los nodos son los valores de re-muestreo. La barra de escala representa la diferencia en nucleótidos de las secuencias. Los números de acceso de GenBank están entre paréntesis. 


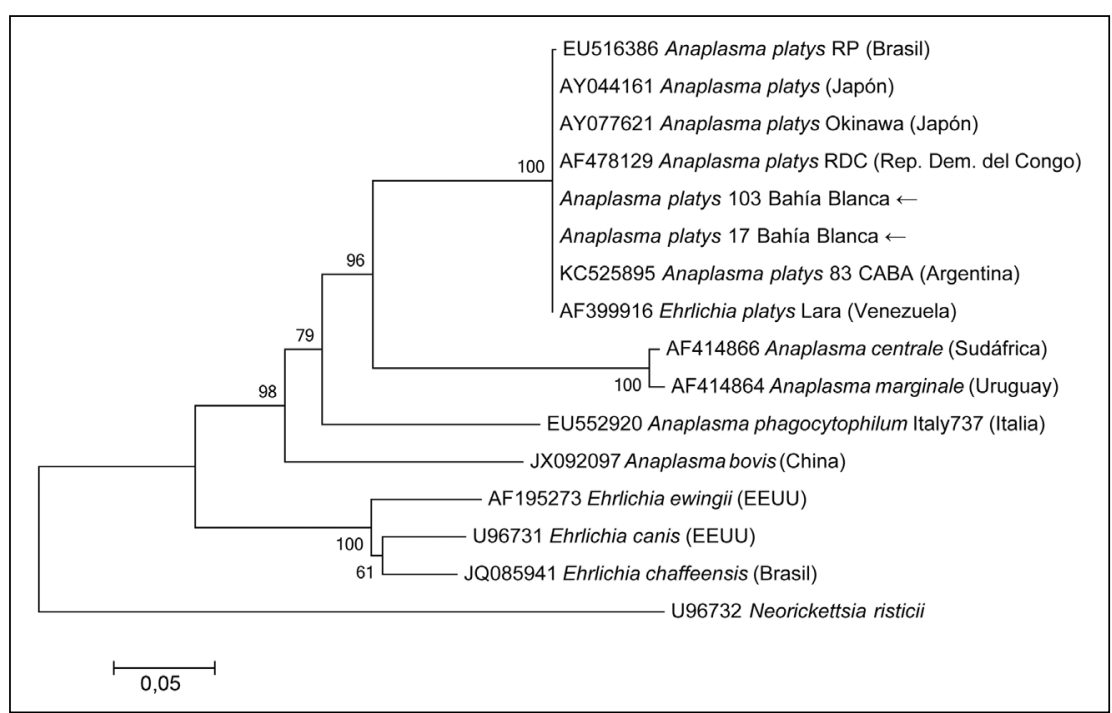

Figura 2. Árbol filogenético inferido por la comparación de secuencias parciales del gen groESL de la familia Anaplasmataceae. Los números en los nodos son los valores de re-muestreo. La barra de escala representa la diferencia en nucleótidos de las secuencias. Los números de acceso del GenBank están entre paréntesis.

\section{Discusión}

Nuestro estudio pone de manifiesto la presencia de $R$. massiliae en garrapatas $R$. sanguineus del Municipio de Bahía Blanca, siendo la segunda zona urbana de Sudamérica donde se ha detectado. En caninos detectamos la infección por A. platys, siendo la región más austral de Argentina donde ha sido notificada.

Rickettsia massiliae ha sido detectada en garrapatas del género Rhipicephalus con diversos niveles de infección, de 2,5-18\% en Europa ${ }^{25,26}, 5-6 \%$ en África ${ }^{27,28}, 25 \%$ en Israel ${ }^{29}$ hasta más de $25 \%$ en Estados Unidos de América, ${ }^{9,30}$. En Sudamérica, sólo había sido detectada en CABA, con un nivel de infección de $3,4^{16}$ a $20 \%{ }^{31}$. Estas diferencias posiblemente se encuentran relacionadas con la población y especies de garrapatas estudiadas (recolectadas de hospederos o de la vegetación) y los métodos diagnósticos utilizados. Además, el nivel de infección puede variar cuando las garrapatas se encuentran ingurgitadas, dado que pueden infectarse con microorganismos circulantes en la sangre del hospedero ${ }^{32}$. Los análisis filogenéticos basados en las secuencias parciales del espacio intergénico 23S-5S ARNr y del gen gltA muestran que todas las muestras positivas tienen $100 \%$ de identidad entre sí, así como con hallazgos previos en Argentina. También presentan un algo grado de identidad con secuencias de R. massiliae previamente reportadas en el mundo.

Por otra parte, previamente se ha detectado seropositividad a Rickettsia spp. en caninos domésticos, aunque, al igual que en nuestro estudio, no se pudo detectar en sangre mediante $\mathrm{RPC}^{9,16,31}$. En caninos, $R$. conorii y $R$. rickettsii producen rickettsemia detectable del día 2 al 12 luego de la infección; sin embargo, no se ha comprobado su susceptibilidad a $R$. massiliae ${ }^{\text {. Por ello, se necesitan }}$ estudios complementarios para dilucidar el rol de los caninos como reservorios de este patógeno.

Anaplasma platys ha sido detectada en caninos domésticos en todo el mundo por RPC, con distintos niveles de prevalencia, de 13,5 a 20\% en América hasta 4 a 9\% en Europa. En Sudamérica fue detectada en Chile en $20 \%{ }^{10}$ y Brasil en $19,9 \%{ }^{12}$ de caninos con signología compatible, y en Venezuela en 16\% de perros con elevado riesgo de infección $^{33}$. En Argentina, ha sido detectada en CABA con una variación de 13,5 a $33 \%$ en caninos clínicamente sanos $^{16}$ y en el lindante Gran Buenos Aires en 15,8\% de caninos con signología clínica compatible ${ }^{15}$. Estas variaciones se deben probablemente a la población canina estudiada (animales clínicamente sanos o con signología compatible), los niveles de infestación por garrapatas y el método diagnóstico utilizado. Los análisis basados en las secuencias parciales del gen 16S ARNr y groESL halladas en nuestro estudio revelaron que las muestras positivas son similares entre sí, así como con hallazgos en Argentina, Chile, Brasil y otras regiones del mundo. Por otra parte, la diferencia hallada en las secuencias del fragmento $16 \mathrm{~S}$ ARNr de un canino con respecto al resto, no se reflejó en el fragmento groESL, quedando por dilucidar la existencia de variaciones genéticas entre estos hallazgos.

La presencia de $A$. platys en garrapatas ha sido escasamente reportada en la literatura científica ${ }^{22,34}$. En Sudamérica sólo fue detectada en garrapatas de un área urbana de Corrientes (noreste de Argentina) ${ }^{35}$. Por otra parte, de los estudios en caninos mencionados en párrafos anteriores, sólo en la investigación realizada en Venezuela se analizaron también las garrapatas, que resultaron todas negativas $^{33}$. En nuestro estudio no detectamos ADN de $A$. platys en las garrapatas analizadas, posiblemente por los diferentes métodos diagnósticos utilizados (por ejemplo, métodos de extracción de $\mathrm{ADN}$, combinación de RPC con hibridación reversa o RPC en tiempo real) y las diferentes poblaciones de garrapatas estudiadas.

Complementariamente, distintos reportes demuestran que $R$. sanguineus está conformado en el cono sur de Sudamérica por dos linajes (norte y sur) con relación filogenética con África y Europa respectivamente, los cuales tendrían diferente competencia vectorial. El linaje norte se comprobó competente como vector en forma experimental y se halló naturalmente infectado con $E$. canis, mientras que el linaje sur no fue competente en forma experimental y tampoco se detectaron ehrlichias en forma natural, conforme con nuestros hallazgos ${ }^{36}$. Por otra parte, $R$. massiliae no fue hallada en los sitios de distribución del linaje norte de $R$. sanguineus en el cono sur de Sudamérica ${ }^{13}$. 
En cuanto a la importancia para la salud pública, la descripción en Argentina de un caso humano debido a $R$. massiliae ${ }^{7}$, así como picaduras de garrapatas a humanos en el área de estudio ${ }^{37}$, destacan la relevancia de este patógeno para la región, especialmente en zonas urbanas ${ }^{8}$. En el caso de $A$. platys, en Venezuela se ha identificado la presencia de mórulas compatibles en el citoplasma de plaquetas de pacientes con seropositividad al virus de la inmunodeficiencia humana ${ }^{38}$ y también se detectó mediante RPC en un veterinario con síndrome febril y co-morbilidades ${ }^{39}$. Sin embargo, se necesitan estudios complementarios para confirmar su rol como agente zoonótico.

En conclusión, los resultados obtenidos en el presente estudio indican la presencia de $R$. massiliae en una nueva zona urbana de Sudamérica, representando un patógeno de importancia en salud pública que se deberá tener presente en los servicios médicos locales. Por otra parte, la detección de $A$. platys se extiende a una ubicación más austral de lo notificado hasta el momento en Argentina. En resumen, estos hallazgos son de importancia para comprender la epidemiología de los patógenos transmitidos por garrapatas en las regiones urbanas y establecer mecanismos adecuados para la prevención de enfermedad humana. Es necesario continuar con la investigación de estos patógenos para ampliar el conocimiento de los reservorios, vectores y ocurrencia de enfermedad humana en distintas áreas urbanas y rurales de la región.

\section{Resumen}

Introducción: Las rickettsiosis, ehrlichiosis y anaplasmosis son causadas por bacterias gramnegativas, intracelulares obligadas y transmitidas principalmente por artrópodos. Objetivo: Detectar y caracterizar molecularmente estos patógenos en garrapatas y caninos domésticos del municipio de Bahía Blanca (provincia de Buenos Aires, Argentina). Material y Métodos: Se estudiaron 56 muestras sanguíneas de caninos, 75 garrapatas Rhipicephalus sanguineus y 7 Amblyomma tigrinum. Las muestras fueron analizadas por RPC para Rickettsia (espacio intergénico 23S-5S ARNr), Ehrlichia y Anaplasma (16S ARNr), y Anaplasma platys (16S ARNr). Resultados: Se detectó positividad a Rickettsia en $12 \%$ de $R$. sanguineus, identificándose por secuenciación a Rickettsia massiliae. Las muestras sanguíneas de los caninos resultaron en $37,5 \%$ positivas a $A$. platys. También se caracterizaron molecularmente por la amplificación del fragmento del gen citrato sintasa (gltA) (género Rickettsia) y del gen groESL (A. platys). Se construyeron árboles filogenéticos utilizando el método del vecino más cercano (neighbor-joining) revelando que las secuencias obtenidas son similares a las de otras regiones geográficas. Conclusión: Los resultados indican la presencia de $R$. massiliae en garrapatas $R$. sanguineus en una segunda zona urbana de Sudamérica y la infección por $A$. platys en caninos, siendo la región más austral de Argentina donde ha sido notificada.

\section{Referencias bibliográficas}

1.- Dumler J S, Barbet A F, Bekker C P, Dasch G A, Palmer G H, Ray S C, et al. Reorganization of genera in the families Rickettsiaceae and Anaplasmataceae in the order Rickettsiales: unification of some species of Ehrlichia with Anaplasma, Cowdria with Ehrlichia and Ehrlichia with Neorickettsia, descriptions of six new species combi. Int J Syst Evol Microbiol 2001; 51: 2145-65.

2.- Parola P, Labruna M B. Tick-borne rickettsioses in America: unanswered questions and emerging diseases. Curr Infect Dis Rep 2009; 11: 40-50.

3.- Raoult D, Roux V. Rickettsioses as paradigms of new or emerging infectious diseases. Clin Microbiol Rev 1997; 10: 694-719.

4.- Cicuttin G. Rickettsiosis urbanas: Rickettsia massiliae. Basualdo Farjat J, Enría D, Martino P, Rosenzvit M, Seijo A (editores). Temas de Zoonosis VI. 1era edición. Buenos Aires: Editorial Ideográfica 2014; 281-6.

5.- Parola P, Socolovschi C, Jeanjean L, Bitam I, Fournier P E, Sotto A, et al. Warmer weather linked to tick attack and emergence of severe rickettsioses. PLoS Negl Trop Dis 2008; 2: e338.

6.- Vitale G, Mansuelo S, Rolain J M, Raoult D. Rickettsia massiliae human isolation. Emerg Infect Dis 2006; 12: 174-5.

7.- García García J C, Portillo A, Núñez M J, Santibáñez S, Castro B, Oteo J. A patient from Argentina infected with Rickettsia massiliae. Am J Trop Med Hyg 2010; 82: 691-2.

8.- Renvoisé A, Delaunay P, Blanchouin E, Cannavo I, Cua E, Socolovschi C, et al. Urban family cluster of spotted fever rickettsiosis linked to Rhipicephalus sanguineus infected with Rickettsia conorii subsp. caspia and Rickettsia massiliae. Ticks Tick Borne Dis 2012; 3: 388-391.

9.- Beeler E, Abramowicz K F, Zambrano M L, Sturgeon M M, Khalaf N, Hu R, et al. A focus of dogs and Rickettsia massiliae-infected Rhipicephalus sanguineus in California. Am J Trop Med Hyg 2011; 84: 244-9.

10.- Abarca K, López J, Perret C, Guerrero J, Godoy P, Veloz A, et al. Anaplasma platys in dogs, Chile. Emerg Infect Dis 2007; 13: 1392-5.

11.- De la Fuente J, Torina A, Naranjo V, Nicosia S,
Alongi A, La Mantia F, et al. Molecular characterization of Anaplasma platys strains from dogs in Sicily, Italy. BMC Vet Res 2006; 2: 24 .

12.- Santos F, Coppede J S, Pereira A L A, Oliveira L P, Roberto P G, Bendetti R B R, et al. Molecular evaluation of the incidence of Ehrlichia canis, Anaplasma platys and Babesia spp. in dogs from Ribeirão Preto, Brazil. Vet J 2009; 179: 145-8.

13.- Ramos C A, Ramos R A N, Araújo F R, Guedes D S, Souza I I F, Ono T M, et al. Comparison of nested-PCR with blood smear examination in detection of Ehrlichia canis and Anaplasma platys in dogs. Rev Bras Parasitol Vet 2009; 18: 58-62.

14.- Venzal D J M, Nava S. El género Rickettsia como agente de zoonosis en el Cono Sur de Sudamérica. Rev Med Urug 2011; 27: 98-106.

15.- Eiras D F, Craviotto M B, Vezzani D, Eyal O, Baneth G. First description of natural Ehrlichia canis and Anaplasma platys infections in dogs from Argentina. Comp Immunol Microbiol Infect Dis 2013; 36: 169-73.

16.- Cicuttin G L, Brambati D F, Rodríguez 
Eugui J I, González Lebrero C, De Salvo M N, Beltrán F J, et al. Molecular characterization of Rickettsia massiliae and Anaplasma platys infecting Rhipicephalus sanguineus ticks and domestic dogs, Buenos Aires (Argentina). Ticks Tick Borne Dis 2014; 5 (5): 484-8. pii: S1877-959X(14)00060-0. doi: 10.1016/j. ttbdis.2014.03.001.

17.- Boero J. Las garrapatas de la República Argentina (Acarina-Ixodoidea). Editorial Universidad de Buenos Aires. Buenos Aires, Argentina. 1957.

18.- Casas I, Powell L, Klapper P, Cleator G. New method for the extraction of viral RNA and DNA from cerebrospinal fluid for use in the polymerase chain reaction assay. J Virol Meth 1995; 53: 25-36.

19.- Jado I, Escudero R, Gil H, Jiménez Alonso M I, Sousa R, García Pérez A L, et al. Molecular method for identification of Rickettsia species in clinical and environmental samples. J Clin Microbiol 2006; 44: 4572-6.

20.- Sousa R, Barata C, Vitorino L, Santos Silva M, Carrapato C, Torgal J, et al. Rickettsia sibirica isolation from a patient and detection in ticks, Portugal. Emerg Infect Dis 2006; 12: 1103-8.

21.- Parola P, Roux V, Camicas J L, Baradji I, Brouqui P, Raoult D. Detection of ehrlichiae in African ticks by polymerase chain reaction. Trans R Soc Trop Med Hyg 2000; 94: 707-9.

22.- Inokuma H, Raoult D, Brouqui P. Detection of Ehrlichia platys DNA in brown dog ticks (Rhipicephalus sanguineus) in Okinawa Island, Japan. J Clin Microbiol 2000; 38: 4219-21.

23.- Inokuma $H$, Fujii K, Okuda M, Onishi T, Beaufils J P, Raoult D, et al. Determination of the nucleotide sequences of heat shock operon groESL and the citrate synthase gene ( gltA) of Anaplasma (Ehrlichia) platys for phylogenetic and diagnostic studies. Clin Diagn Lab Immunol 2002; 9: 1132-6.

24.- Tamura K, Peterson D, Peterson N, Stecher G, Nei M, Kumar S. MEGA5: Molecular evolutionary genetics analysis using maximum likelihood, evolutionary distance, and maximum parsimony methods. Mol Biol Evol 2011; 28: 2731-9.

25.- Márquez F J, Rodríguez Liébana J J, Soriguer R C, Muniaín M A, Bernabeu Wittel M, Caruz A, et al. Spotted fever group Rickettsia in brown dog ticks Rhipicephalus sanguineus in southwestern Spain. Parasitol Res 2008; 103: 119-22.

26.- Psaroulaki A, Spyridaki I, Ioannidis A, Babalis T, Gikas A, Tselentis Y. First isolation and identification of Rickettsia conorii from ticks collected in the region of Fokida in Central Greece. J Clin Microbiol 2003; 41: 3317-9.

27.- Sarih M, Socolovschi C, Boudebouch N, Hassar M, Raoult D, Parola P. Spotted fever group rickettsiae in ticks, Morocco. Emerg Infect Dis 2008; 14: 1067-73.

28.- Khaldi M, Socolovschi C, Benyettou M, Barech G, Biche M, Kernif T, et al. Rickettsiae in arthropods collected from the north African hedgehog (Atelerix algirus) and the desert hedgehog (Paraechinus aethiopicus) in Algeria Comp Immunol Microbiol Infect Dis 2012; 35: 117-22.

29.- Harrus S, Perlman Avrahami A, Mumcuoglu K Y, Morick D, Baneth G. Molecular detection of Rickettsia massiliae, Rickettsia sibirica mongolitimonae and Rickettsia conorii israelensis in ticks from Israel. Clin Microbiol Infect 2011; 17: 176-80.

30.- Eremeeva M E, Bosserman E A, Demma L J, Zambrano M L, Blau D M, Dasch G A. Isolation and identification of Rickettsia massiliae from Rhipicephalus sanguineus ticks collected in Arizona. Appl Environ Microbiol 2006; 72: 5569-77.

31.- Cicuttin G L, Rodríguez Vargas M, Jado I, Anda P. Primera detección de Rickettsia massiliae en la Ciudad de Buenos Aires. Rev Arg Zoon 2004; I: 8-10.

32.- Estrada Peña A, Gray J S, Kahl O, Lane R S, Nijhof A M. Research on the ecology of ticks and tick-borne pathogens methodological principles and caveats. Front Cell Infect Microbiol 2013; 3: 29.

33.- Huang H, Unver A, Pérez M J, Orellana N G, Rikihisa Y. Prevalence and molecular analysis of Anaplasma platys in dogs in Lara, Venezuela. Braz J Microbiol 2005; 36: 211-6.

34.- Sanogo Y O, Davoust B, Inokuma H, Camicas J L, Parola P, Brouqui P. First evidence of Anaplasma platys in Rhipicephalus sanguineus (Acari: Ixodida) collected from dogs in Africa. Onderstepoort J Vet Res 2003; 70 : 205-12.

35.- Oscherov E B, Milano A M F, Lobo B, Anda P, Escudero R. Detection of Anaplasma platys and other pathogens in ectoparasites from urban hosts in Northeast Argentine. Rev Ibero-Latinoam Parasitol 2011; 70: 42-7.

36.- Nava S, Mastropaolo M, Venzal J M, Mangold A J, Guglielmone A A. Mitochondrial DNA analysis of Rhipicephalus sanguineus sensu lato (Acari: Ixodidae) in the Southern Cone of South America. Vet Parasitol 2012; 190: 547-55.

37.- Cicuttin G L, Brambati D F, Rodríguez Eugui J I, González Lebrero C, De Salvo M N, Vidal P, et al. Garrapatas duras (Familia Ixodidae) en caninos domésticos de la Ciudad Autónoma de Buenos Aires y del municipio de Bahía Blanca (Argentina). En: 2do Encuentro Nacional sobre Enfermedades Olvidadas y XIV Simposio Internacional sobre Control Epidemiológico de Enfermedades Transmitidas por Vectores. Buenos Aires, Argentina. 2011.

38.- Tamí C D, Tamí I. Identificación morfológica de Ehrlichia sp. en las plaquetas de pacientes con infección por virus de la inmunodeficiencia humana en Venezuela. Rev Panam Salud Publica 2004; 16: 345-9.

39.- Maggi R G, Mascarelli P E, Havenga L N, Naidoo V, Breitschwerdt E B. Co-infection with Anaplasma platys, Bartonella henselae and Candidatus Mycoplasma haematoparvum in a veterinarian. Parasit Vectors 2013; 6: 103. 